\title{
Motologische und arbeitswissenschaftliche
}

\section{Ansätze für die Gesundheitsförderung im Setting} »Industrie»

Ergänzend zu dem Beitrag "Gesundheitsförderung von Produktionsmitarbeitern - Synergien aus Motologie und Arbeitswissenschaft « in diesem Heft werden Methoden zur Analyse und Bewertung der Arbeitsbedingungen, der individuellen Befindlichkeit des Mitarbeiters sowie Maßnahmen zur Gesundheitsförderung an Produktionsarbeitsplätzen aufgezeigt. Um die Gesundheit und Arbeitsfähigkeit der Mitarbeiter zu erhalten, ist es von Bedeutung, zum einen die Belastungen in den Produktionsbereichen zu optimieren und zum anderen diese auf die Fähigkeiten der Mitarbeiter hin anzupassen. Zusätzlich ist die individuelle Perspektive auf die Gesundheit eines Mitarbeiters wichtig, der mit paradoxen Erfahrungen und konträren Interessenkonflikten durch die Entstandardisierung und Enttraditionalisierung in der postmodernen Zeit konfrontiert ist (Schröder 2009).

\section{Von Risikoreduktion bis Ressourcenstärkung}

\section{Fallbeispiel}

Herr Müller arbeitet seit 15 Jahren in einer Firma zur Reifeninstandhaltung. Seit dem vergangenen Jahr klagt Herr Müller vermehrt über plötzlich auftretende Schmer-

\begin{abstract}
zen im unteren Rücken. Vor kurzem ist ihm der Schmerz wie ein Blitz ins Kreuz gefahren, als er im Lager einen Reifen aus dem Regel wuchtete und mit einer schnellen Oberkörperdrehung auf den Boden gesetzt hat. Die Weiterführung dieser Tätigkeit in der Firma erfüllt Herrn Müller mit großer Sorge.
\end{abstract}

Arbeitsplatzbewertungen mithilfe von Screeningverfahren stellen Maßnahmen zur Erfassung der Ist-Situation aufseiten der verhältnisorientierten Maßnahmen dar und dienen der Schaffung menschengerechter Arbeitsbedingungen. Hohe Belastungen können so ermittelt und ergonomische Gestaltungslösungen abgeleitet werden. Hierfür stehen aus der Arbeitswissenschaft verschiedene Ergonomie-Bewertungsmethoden zur Verfügung, die sich auf wissenschaftliche Erkenntnisse und gängige Normen stützen. Ein in Deutschland weiterverbreitetes Experten-Screening sind z.B. das Ergonomic Assessment Worksheet (EAWS, Schaub et al. 2012) oder die Leitmerkmalmethoden der Bundesanstalt für Arbeitsschutz und Arbeitsmedizin BAuA (2012) als Grob-Screening. Hierbei werden körperliche Belastungen erfasst, z. B. hohe Lastenhandhabung, und mit einem Punktwert versehen. Der Ge- samtpunktwert gibt Aufschluss darüber, wie hoch die gesundheitliche $\mathrm{Ge}$ fährdung an dem Arbeitsplatz ist. Bei der Arbeitsplatzumgestaltung ist es wichtig, die Mitarbeiter mit einzubeziehen, um eine Akzeptanz des neu gestalteten Arbeitsplatzes zu erreichen. Die Mitarbeiter werden durch Befragungen oder weitere Untersuchungen wie z.B. Muskelaktivitätsmessungen (EMG) oder Herzschlagfrequenzmessungen am Arbeitsplatz sowie bei einer evtl. stattfindenden Abschlussevaluation miteinbezogen. Bei der Befragung werden beispielsweise der Fragebogen zur subjektiven Einschätzung der Belastung am Arbeitsplatz (FEBA, Slesina 1987), das Beanspruchungsempfinden mittels der Borg-Skala (Borg 2004) oder ein Fragebogen zu Beschwerden am Bewegungsapparat wie die Body Part Discomfort Scale (Corlett/ Bishop 1976) herangezogen. Dabei bestehen große Gemeinsamkeiten zu dem motologischen Gesundheitsmapping von Schröder/Köhnen (2009). Das Gesundheitsmapping besteht aus dem Körper-Mapping (Visualisierung der Beschwerden am Körper), Mapping der Gesundheitsrisiken am Arbeitsplatz (Visualisierung der Probleme am Arbeitsplatz) und "Deine Welt«-Mapping (Visualisierung der Auswirkungen auf das eigene Leben). Danach werden 
eine Prioritätenliste und ein Aktionsplan aufgestellt, um lösungsorientierte Aktionen zu planen. Ziel ist es, dadurch vor allem die subjektive Sichtweise der Beschäftigten wie z.B. Arbeitserfahrungen, die eigenen leiblichen Befindlichkeiten und das eigene Verhalten im Kontext der betrieblichen Situation sowie den Umgang mit Kollegen und das Verhalten im außerbetrieblichen Alltag zu berücksichtigen (Schröder/Köhnen 2009).

Die Arbeitswissenschaft nutzt die Untersuchungsergebnisse, um Belastungsschwerpunkte abzuleiten, aus denen im nächsten Schritt Gestaltungslösungen generiert werden. Neben technischen Gestaltungslösungen (d.h. Verringerung von körperlichen Belastungen am Arbeitsplatz durch Gestaltungslösungen) werden auch organisatorische (z.B. Schichtpläne) und persönliche Maßnahmen (ErgonomieSchulungen am Arbeitsplatz) angewandt.

Die motologische Kompetenz über Bewegung und Körperlichkeit könnte bei der Optimierung der körperlichen Bewegungsabläufe und der Reduzierung von physischen Belastungen eine sinnvolle Ergänzung darstellen. Mit dem Wissen aus der Organisationsberatung könnte eine Unterstützung bei den organisatorischen Gestaltungslösungen stattfinden und die motologische Expertise zu den individuellen Maßnahmen eingebracht werden. Besonders die Verhaltensorientierung mit individuellen Maßnahmen könnte auf Basis des Gesundheitsmappings oder durch die Implementierung von Fragebögen wie den Frankfurter Körperund Selbstkonzept-Skalen nach Deusinger (1998) und dem Fragebogen zu krankheitsbezogenen Kontrollüberzeugungen (Lohhaus/Schmidt 1989) bereichert werden. Inhalte der motologischen Intervention könnten hierbei sein: leibliche Sensibilitäten bewusstmachen

- eigene Einstellung besser reflektieren wie z. B. Kontrollüberzeugungen oder Überidentifikationen (Schröder / Köhnen 2009)

Dies kann dabei helfen, die richtige Distanz zum eigenen Tun zu finden und die Entscheidungsfindung zu unterstützen. Weitere Interventionsmöglichkeiten liegen im Aufbau psychosozialer Ressourcen wie Selbstwirksamkeit, Stimmungsbeeinflussung, Körpergefühl und Körperkonzept, positiven Bewegungserlebnissen über Körpererfahrung, Unterstützung der Selbstsorge (Haas et al. 2007).

Mögliche motologische und arbeitswissenschaftliche Maßnahmen bezogen auf das Fallbeispiel können sein:

\section{Technische Maßnahmen}

- Transport-/Hebehilfen bzw. Greifhilfen bereitstellen und regelmäßig warten,

- Platzierung der Räder auf optimaler Höhe

\section{Organisatorische Maßnahmen}

- Organisation von Wechseltätigkeiten / Rotation am Arbeitsplatz,

- Unterstützung durch zweiten Mitarbeiter

\section{Persönliche Maßnahmen}

arbeitsmedizinische Untersuchung des Rückens,

- verhaltensorientierte Maßnahmen wie arbeitsplatzbezogene Rückenschule, psychomotorische Therapie, Erlernen von aktiven Entspannungstechniken etc.

Um eine korrektive Maßnahmen zu vermeiden, ist es bereits sinnvoll, pro- spektive Gesundheitsförderung zu praktizieren und Arbeitsplätze und Arbeitsbedingungen so zu planen, dass die Gesundheitsrisiken gering sind. Ein Instrument hierfür bietet die sog. Fähigkeitsdatenbank, die für Arbeitsplatzplaner in der Automobilindustrie entwickelt wurde, jedoch auch zur motologischen Gesundheitsförderung genutzt werden kann (Müglich et al. 2015). Die Datenbank enthält anonymisierte Fähigkeitsprofile, die von Arbeitsmedizinern erstellt wurden, um die körperliche Leistungsfähigkeit der Produktionsmitarbeiter eines großen deutschen Automobilkonzerns zu ermitteln. Eine Funktion der Datenbank besteht darin, sog. »kritische« Fähigkeiten zu identifizieren, die mind. $20 \%$ der Mitarbeiter nicht mehr ausführen können. Zu den kritischen Fähigkeiten zählen beispielsweise:

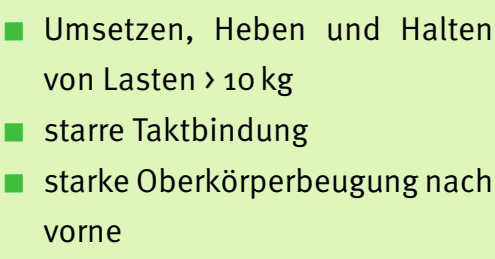

Bei den individuellen Maßnahmen bzw. den Maßnahmen der psychomotorischen Gruppentherapie, der medizinischen Trainingstherapie oder der Vermittlung von Entspannungstechniken wie von Haas et al. (2007) beschrieben, könnte sich die motologische Intervention auf diese »kritischen« Fähigkeiten fokussieren. Ein Ansatz besteht in der Stärkung physischer Ressourcen, wobei diese Fähigkeiten und der Umgang mit den körperlichen Belastungssituationen gezielt trainiert werden. Ein weiterer Ansatz könnte versuchen, über die Förderung der Entspannungsfähigkeit einen Ausgleich zu den Belastungssituationen zu schaffen.

\section{Dorothee Müglich}

DOI 10.2378 / motorik2017.art15d 


\section{Literatur}

Borg G. (2004): Anstrengungsempfinden und körperliche Aktivität. Deutsches Ärzteblatt 101 (15), A-1016-1021

Bundesanstalt für Arbeitsschutz und Arbeitsmedizin (BAuA) (2012):

Gefährdungsbeurteilung mithilfe der Leitmerkmalmethode. In: http:// www.baua.de/de/Themen-von-A-Z/ Physische-Belastung/Gefaehrdungs beurteilung.html, 13.12.2016

Corlett, E.N., Bishop, R.P. (1976): A technique for measuring postural discom fort. Ergonomics 41 (9), 175-182

Deusinger, I.M. (1998): Frankfurter Körperkonzeptskalen (FKKS) - Handanweisung. Hogrefe-Verlag, Göttingen

Haas, R., Ohlenburg, H., Glauninger, B., Jakobs, S., Tiemann, H. (2007): Psy- chomotorische Begleitung als ein Baustein eines methodenintegrativen Angebots für Menschen mit bio-psycho-sozialen Gesundheitsproblemen in der industriellen Fertigung. motorik 30 (3), 157-164

Lohhaus, A., Schmitt, G.M. (1989): Fragebogen zur Erhebung von Kontrollüberzeugungen zu Krankheit und Gesundheit (KKG) - Handanweisung. Hogrefe-Verlag, Göttingen

Müglich, D., Sinn-Behrendt A., Schaub K., Bruder R. (2015): Development of a database for capability-appropriate workplace design in manufacturing industry. Occupational Ergonomics 12 (3), 109-118, http://dx.doi. org/10.3233/OER-150228

Schröder, J. (2009): Besinnung in flexiblen Zeiten - Leibliche Perspek- tiven auf postmoderne Arbeit. VSVerlag, Wiesbaden, http://dx.doi. org/10.1007/978-3-531-91866-2

Schröder, J., Köhnen, H. (2009): Gesundheitsförderliche Organisationsentwicklung durch Gesundheitsmapping. motorik 32 (1), 17-23

Schaub, K., Caragnano, G., Britzke, B. Bruder, R. (2012): The European As sembly Worksheet. Theoretical Issues in Ergonomics Science 14 (6), 616639

Slesina, W. (1987): Arbeitsbedingte Erkrankungen und Arbeitsanalyse - Arbeitsanalyse unter dem Gesichtspunkt der Gesundheitsvorsorge. Enke, Stuttgart

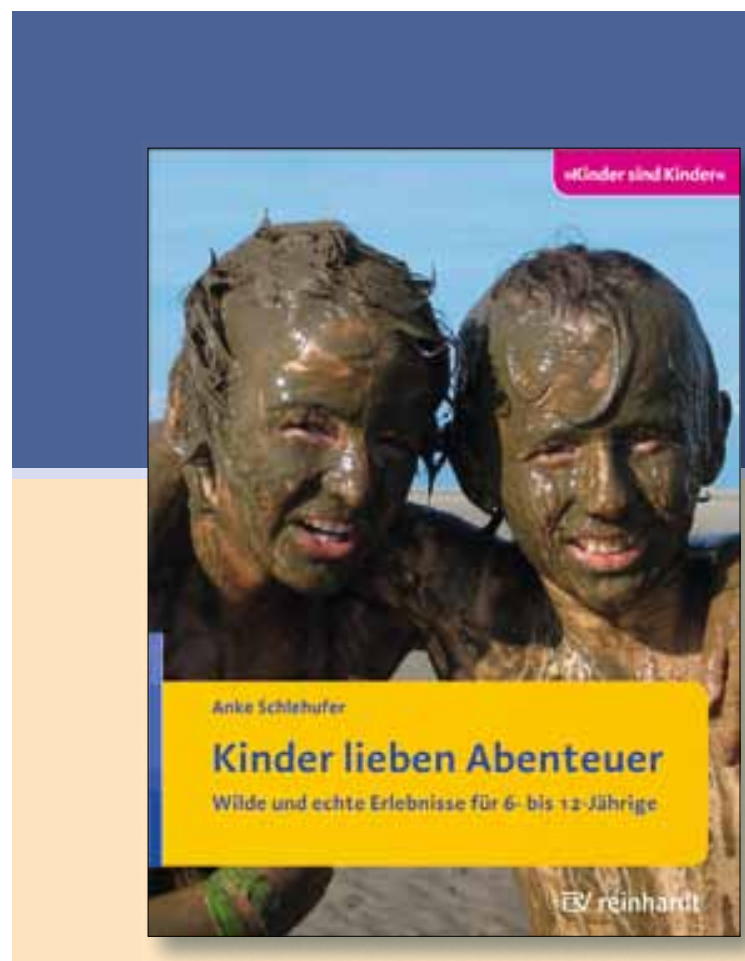

2016. 160 Seiten. Mit 41 Fotos. Innenteil zweifarbig.

(978-3-497-02543-5) kt

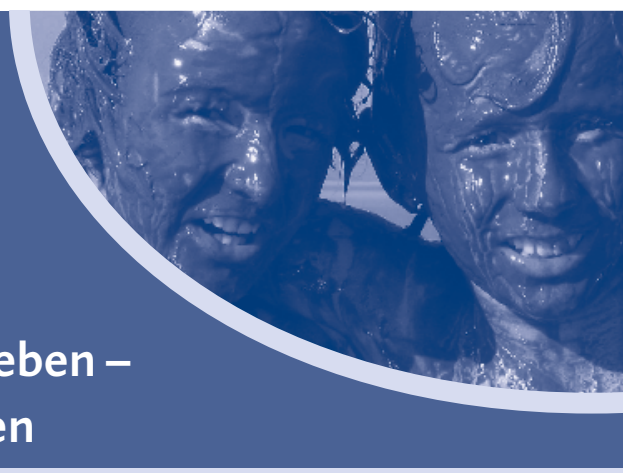

Kinder im Alter von 6 bis 12 Jahren wollen sich selbst und die Welt erforschen und begreifen. Wenn sie Abenteuer erleben, fühlen sie sich lebendig, lernen Ausdauer und Zuversicht, stillen ihre Neugier und ihren Entdeckerdrang. Sie spüren, dass sie allein und mit anderen etwas bewirken können

Wie können Eltern Kinder in ihrer Abenteuerlust und ihrem Bedürfnis nach Autonomie, freiem Spiel und Naturerfahrung unterstützen? In diesem Buch finden sie eine Fülle von praktischen Anregungen - für eine Vielfalt von Abenteuern rund um die Themen der Kindheit: Natur, Elemente, Jahreszeiten, Bewegung, Freundschaft, Fantasie, Tiere, Bauen, Sammeln, Forschen ...
EV reinhardt

www.reinhardt-verlag.de 